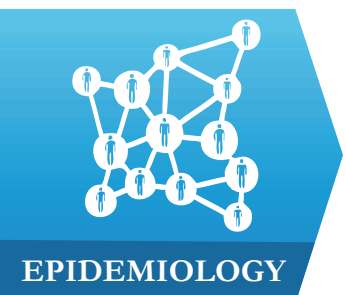

1) Iuliu Hatieganu University of Medicine and Pharmacy, Cluj-Napoca, Romania

2) Department of Pathology, "Prof. Dr. Ion Chiricuta" Oncology Institute, Cluj-Napoca, Romania

3) Department of Information Technology, "Prof. Dr. Ion Chiricuta" Oncology Institute, Cluj-Napoca ClujNapoca, Romania

4) Department of Medical Genetics, Iuliu Hatieganu University of Medicine and Pharmacy, Cluj-Napoca, Romania

5) Department of Genetic Explorations, "Prof. Dr. Ion Chiricuta" Oncology Institute, Cluj-Napoca ClujNapoca, Romania

6) Department of Surgical and Gynecological Oncology, Iuliu Hatieganu University of Medicine and Pharmacy, Cluj-Napoca, Romania

7) Department of Surgery, "Prof. Dr. Ion Chiricuta" Oncology Institute, Cluj-Napoca, Romania

8) Department of Medical Informatics and Biostatistics, Iuliu Hatieganu University of Medicine and Pharmacy, Cluj-Napoca, Romania

9) Department of Internal Medicine, "Prof. Dr. Ion Chiricuta" Oncology Institute, Cluj-Napoca Cluj-Napoca, Romania

DOI: $10.15386 /$ cjmed-1015

Manuscript received: 13.03.2018

Received in revised form: 19.07.2018 Accepted: 23.07.2018

Address for correspondence: pop.bogdan21@gmail.com

\title{
The role of medical registries, potential applications and limitations
}

Bogdan Pop ${ }^{1,2}$, Bogdan Fetica ${ }^{2}$, Mihaiela Luminita Blaga ${ }^{3}$,

Adrian Pavel Trifa ${ }^{4,5}$, Patriciu Achimas-Cadariu ${ }^{6,7}$, Catalin Ioan Vlad ${ }^{6,7}$, Andrei Achimas-Cadariu ${ }^{8}$

\begin{abstract}
Medical registries provide highly reliable data, challenged hierarchically only by randomized controlled trials. Although registries have been used in several fields of medicine for more than a century and a half, their key role is frequently overlooked and poorly recognized. Medical registries have evolved from calculating basic epidemiological data (incidence, prevalence, mortality) to diverse applications in disease prevention, early diagnosis and screening programs, treatment response, health care planning, decision making and disease control programs. Implementing, maintaining and running a medical registry requires substantial effort. Developing the registry represents a complex task and is one of the major barriers in widespread use of registries. Medical registries have potential to evolve to a next generation by taking benefit from recent semantic web technology developments. This paper is aimed at providing a summary of the basic information available on medical registries and to highlight the progress and potential applications in this field.
\end{abstract}

Keywords: registries, data accuracy, quality assurance, health care, registries/ standards, public health

\section{Introduction}

David Solomon defines medical registries as "a data base of identifiable persons containing a clearly defined set of health and demographic data collected for a specific public health purpose" [1]. Modern medical registries have initially proven their utility by producing valuable epidemiological data that allowed patient and diseases surveillance [2,3]. Further developments in the field have allowed their use in several domains from population-based studies on medical care, services and technology, early diagnosis and risk group monitoring to health care planning and identifying areas in health care services policies that require intervention $[2,4,5]$. Furthermore in some medical fields registries are essential parts of disease control programs [4]. Linkage of registry information with other data collections like biobanks and the use of registry data in randomized controlled trials will allow the development of populationbased studies in several fields [6]. Although registries have been used in several fields of medicine for more than a century and a half, their significant role is frequently overlooked and poorly recognized $[7,8]$. This paper is aimed at providing a summary of the basic information available on medical registries and to highlight the progress and potential applications in this field.

\section{Medical registries. The basics}

The main uses of medical registries include patient care, public health, technology assessment and research [9]. Patient care applications are variable and include improvement of care quality, evaluation of patient outcome and determining treatment effectiveness, providing information on the natural history of disease, active monitoring of risk groups and providing risk stratification $[2,9]$. In public health, registry data contribute to population surveillance, assessment of disease burden in the general population, health care planning and identifying areas in health care services policies that require intervention $[2,9]$. Technology assessment of medical devices or biopharmaceutical products allow authorities to ensure their safe usage by determining effectiveness and measuring and monitoring unexpected or harmful events [2]. Registries contribute to research by providing resources for observational studies, improving research 
design, studies of processes and hypothesis testing $[2,10]$.

But how do medical registries accomplish such a wide range of functions and what makes them able to generate such reliable data in comparison to other clinical data collection tools? This is achieved by using a combination of predefined data collection protocols, accessing multiple data sources and collecting the highest possible number of cases from a geographically defined population, doubled by explicit efforts to provide systematic constant data update $[2,9,11]$.

\section{Defining characteristics, data sources and classification of registries}

Before discussing data sources another warranted discussion issue is represented by the distinction of medical registries from other medical data collections [1]. Donaldson formulated four characteristics of medical registers that separate them from other medical data collections: medical registers are based on people not events; the registered persons share a common feature, overlapping the registry's purpose; the information contained in registers is updated periodically, in a predefined, systematic manner and finally they are based on a geographically defined population $[9,11]$.

Most databases are hosted and collect data (usually) from a single medical institution, whereas with registries the collection of data is typically performed from multiple institutions [9]. For clinical databases, the definition of common features is not generally mandatory, while for registries, it represents a requirement [9]. In the case of registries explicit efforts are made for the collection of the highest number of cases (in total if possible), making the registries representative data sources for a defined population $[2,9,12]$. Clinical databases rely on the cases presented in an institution and no supplementary efforts are made for the gathering of new cases $[2,9,12]$. Furthermore, in the case of registries data there is a constant update of the patient status, throughout a predefined, well established schedule [9]. Clinical databases are central data repositories that can serve organizational, administrative or research purposes and usually contain data from several sources, which can include individual Electronic Health Records (EHRs). The main distinction between EHRs and clinical databases is represented by the fact that a clinical database usually contains data collected from several patients, while an EHRs contains data from a single individual [2]. The interactions between EHRs and clinical databases are complex and are subjected to variations according to individual organization schematics of the institutions data system. Data access for EHRs are limited to internal personnel involved in current medical practice and can be limited, for example, for research personnel. Clinical databases and EHR represent data sources for registries (Figure 1). EHRs contain all the personal health information of an individuals, across their lifetime, which includes all presentation records in health care facilities [13]. EHRs comply to individual states standards that allow interoperability which allow medical staff in several institutions to record and access patient data [2]. The data in medical registries are focused on allowing results comparison that extend in many instances national borders. EHRs are designed to collect and use data for individual patient benefits. The data collected in medical registries, on the other hand, is driven by the purpose of the registry and the specified outcomes [2]. In contrast to EHRs, for which the collected data are highly variable, according to the individual's medical history, registries collect uniform data, for each individual, in every data collection location, by following a predefined procedure. EHRs are focused on individuals and medical registries are focused on populations $[2,9]$.

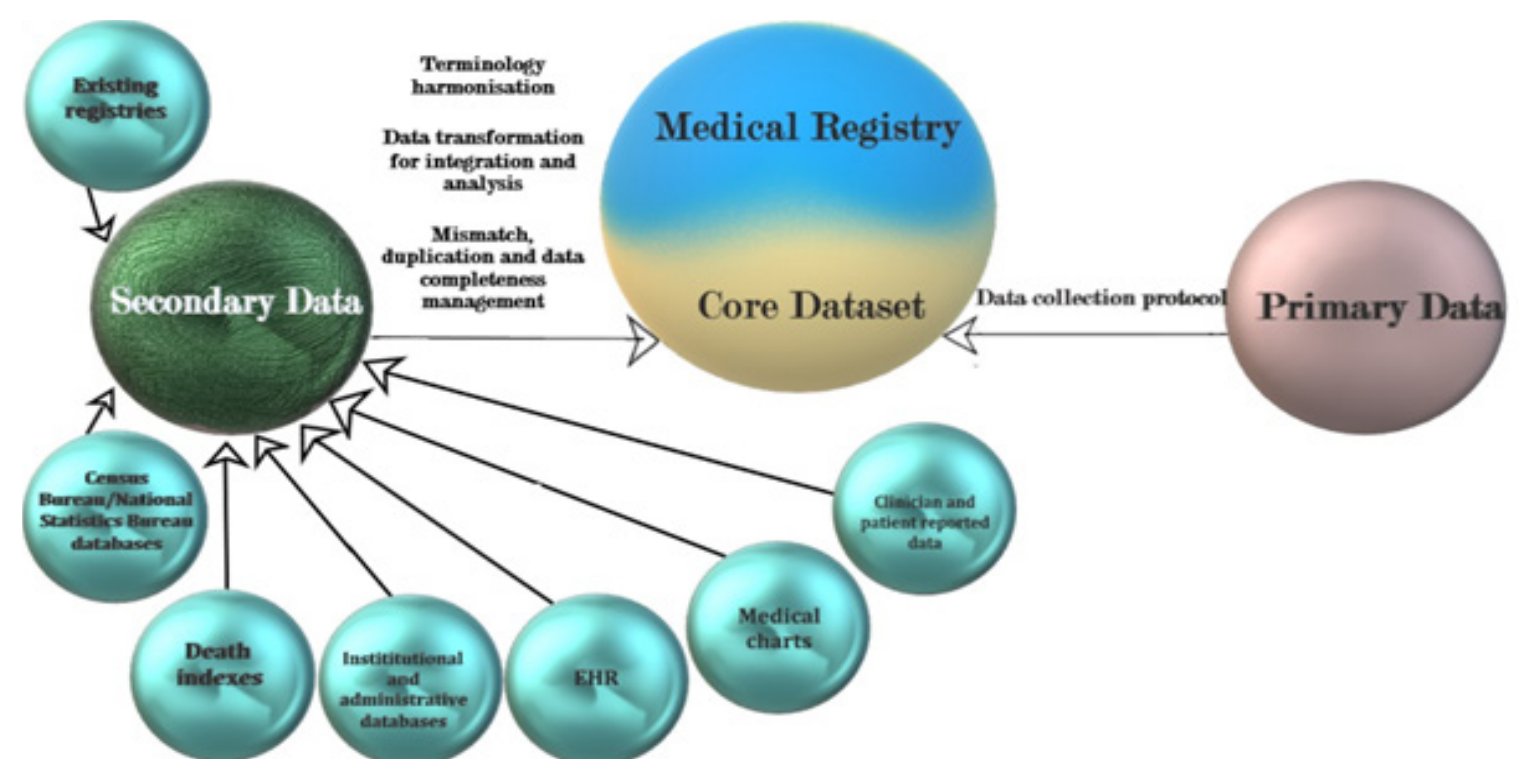

Figure 1. Data sources for medical registries and their relationship with the core dataset [2]. 
Several registries classifications system have been used over time [14-18]. Gliklich proposed a classification based on the registry population definition and identified three main categories: product registries (patient exposed to health care products), health services registries (patient exposed to health care service) and disease and condition registries (state of disease or condition dictates inclusion) [2]. Pedersen, when referring to cancer registries, classified them according to the population covered by data collection [19]. The categories resulting were: local hospital registries (serving one hospital or medical facility), central registries (collect data from several selected hospitals within a region) and population-based registries (which attempt to collect detailed information on all cases suffering from the studied disease in a population of known size and composition) [9,19]. A similar modified approach to registries classification is used by the International Epidemiological Association [9].

\section{Challenges in widespread use of medical registries}

Implementing, maintaining and running a medical registry requires substantial effort. Developing the registry represents a complex task and is one of the major barriers in widespread use of registries. When formulating the purpose of the registry, one should balance the requirements and amount of work required for achieving the objective to the available resources [2]. In other words, prior to implementing a registry one needs to balance funding requirements and coverage to the stated purpose and objectives. A successful registry team requires involvement of all the key stakeholders from different areas of expertise and to group them in hierarchical structures, which cover a vast array of functions like data collection, data usage and results dissemination [2]. The registry functionality is determined by proper definition of interactions and communication between stakeholders and their proportional involvement in the decision-making process [2]. Clearly formulated objectives, inclusion criteria, patient outcomes, procedures that cover all the activities of the registry and a properly defined core dataset are mandatory requirements that ensure the fulfillment of the purpose and objectives [2].

The "survival" of the medical registry is dependent on a constant financial support, which according to the registries purpose may be substantial [15]. For example, in the European Union (EU), the most important issue that limits the sustainability of medical registries is represented by unstable funding [15].

Another important requirement for medical registries is represented by mandatory alignment to the legal framework [15]. The most important law that has an impact on medical registries in the EU was until 2016, the Data Protection Directive (95/46/EC), and was replaced by the Regulation 679/2016 (EU), the General Data
Protection Regulation, applicable since May 25 $5^{\text {th }}, 2018$ $[15,20]$. Furthermore, the registration activity is subjected to National Legal Frameworks in Member States which are still being adapted and subject to harmonization across the EU [15]. Beyond registration activity, the matter of data ownership is another legal very complex issue and most often require expert input [15,21,22].

Failure to properly involve stakeholders, a major source for data gathering and results dissemination, will result into unclear stakeholder roles and most likely will have a major impact on the registry outcome $[2,15]$. Furthermore, unclear stakeholder's role can translate into an inadequate transparency and insufficient data dissemination [15].

The lack of knowledge and implementation of current standards and guidelines in clinical practice and registration activity impacts the definition of datasets and the quality of the registry's results [15].

The infrastructure required for the registration activity can also represent a challenge. It includes hardware, software, IT support and personnel training or hardware upgrades that may be necessary over time $[9,23]$. The usage of a software systems reduces the volume of inaccurate information compared to handwritten data-forms that some registries use [15,22].

When compared to clinical trials, registries have several shortcomings. Levine and Julian identified four main limitations of patient registries when compared to clinical trials: patients are not randomly allocated to the intervention for registries, patients follow-up is more active and standardized for randomized trials, missing or incomplete data may represent a greater source of bias for registries and the registry enrollment is less supervised compared with randomized trials [24].

\section{Assessing the quality of registry data and registry results}

Due to their extended range of use and variations in implementations, agreement on quality assessment for medical registries is still a matter of debate [2]. In recent years, several guidelines and recommendations on registry development, implementation, operation and evaluation have been published $[2,25,26]$. Following a literature review, Zaletel et al defined four linked groups of "quality influencing factors": governance; data quality; information and ethical issues; and security and privacy [15]. In a review of literature Arts et al identified and defined two main data quality attributes "accuracy" and "completeness" and formulated literature-based definitions for them [27]. Data accuracy according to Arts represent "the extent to which registered data are in conformity to the truth" [27]. Data completeness is defined by Arts as "the extent to which all necessary data that could have been registered have actually been registered" [27]. 
Bray and Parkin, when referring to cancer registries, formulated four key aspects of data quality: comparability, validity, timeliness and completeness [28,29]. The use of standardized classification and coding systems and consistent definitions will allow the comparison of the registry results between populations, over time [26]. The ICD-O codes represent standards for classification and coding used by cancer registries [26]. Validity is assessed using several numerical indices like the size of bias [28]. Most frequently reported statistics that impact data accuracy are the percentage of cases with missing data, the percentage of cases with a morphologically verified diagnosis and the percentage of cases for with information limited to death certificates [26]. Timeliness vary greatly for cancer registries according to the ability the registry can collect, process, and report data [26]. Data completeness can be assessed by using qualitative (assessing the degree of completeness relative to other registries, or over time) and/or quantitative methods (that provide a numerical evaluation of the extent to which all eligible cases have been registered) [26,29].

Data errors in clinical research databases and registries have been classified in several diverse ways [30]. A study by van der Putten et al identified three main factors that cause errors: mistakes in interpretation, documentation, and coding [30,31]. Sorensen classified data errors into systematic (type I, that impact a large number of data errors and can have a serious impact on data validity, programming errors or data definition errors and data collection protocols errors) and random errors (type II, data recording, transferring of data interpretation errors, with less extent, but more difficult to identify) $[27,30,32]$.

Registry results can be assessed in several ways. Pryor et al described factors that predict the registry success: appropriate multidisciplinary team; stable funding; focused aims; data collection systems and design that relate well to function; and relevant leadership [9,10]. Drolet and Johnson, following an extensive review of peer-reviewed publications focused on registries, formulated five distinguishing features, that allow the highest registry functionality: mergeable data, standardized datasets, rules for data collection, observations associated over time and knowledge about patient outcomes [33]. Medical registries provide highly reliable data, challenged hierarchically only by randomized controlled trials [2]. Scientific publications that result from registry activity can represent partially quantifiable means of assessing the value of registry information, by following several indicators such as number of publications, frequency, topicality, impact factor and citation index [15]. One filter for quality assessment is the use of peer-reviewed scientific publications. Levine and Julian, in an editorial, published a list of criteria that readers are encouraged to use when assessing a registry publication [24]. Other purpose derived quality measures include usage of registry statistical data in policy making, management and improving treatment outcomes [15].

\section{Examples of medical fields benefiting from registration activity}

The wide range of uses for medical registries implies that most medical fields benefit in some way from registries results. The examples provided here are by no means exhaustive, and they more likely represent medical fields that historically have benefited from the registry information.

One of the first domains in which medical registries have been found to be extremely useful is the field of chronic infectious disease [9]. As a matter of fact, the National Leprosy Registry of Norway is considered to be the first modern registry, founded following the Royal Decree of $1856[8,34,35]$. Other examples of historical important uses of patient registries in the field of chronic infectious disease include the study of tuberculosis [36-39]. In fact, in 2012 data submitted to the World Health Organization (WHO) was received from the registration systems from 126 countries $[39,40]$.

The study of rare disease has also benefited from the uses of registries. The ORPHANET initiative, a portal led by a consortium of 40 countries and coordinated by a French team, has published in 2017 a list of rare diseases and orphan drugs registers available in the European Union and neighboring countries, including a number of 703 registers, of which 496 have national coverage [41]. Romania contributes with two disease registries: the Romanian biliary atresia registry and the Romanian cystic fibrosis patient registry [41].

The study of chronic non-communicable disease has benefited also from patient registration. For example, diabetes mellitus registries are used globally in about $44 \%$ of countries [42]. The study of neoplasia has historically benefited from registries results. In effect, 290 registries from 68 countries contributed to the data compiled in the volume $\mathrm{X}$ of the Cancer Incidence in Five Continents $[43,44]$. Cancer registration is being performed for almost a century now. Modern cancer registration was historically started in 1926 with the implementation the cancer registry in Hamburg [4,45]. Earlier attempts for cancer registrations have been recorded [9]. Around the same time, in several locations, similar cancer registration efforts have been set in motion (England (1930), United States of America (1927, 1940), France (1942), Spain (1960), Hungary (1952), Norway (1952), Finland (1952), Sweden (1958), Denmark (1942), Iceland (1953) etc.) [4,9,4648]. Multinational initiatives were set up in the 1950 s, following the establishment of the WHO subcommittee for cancer registration, which provided the first set of methodological guidelines for cancer registration $[4,49]$. Although substantial efforts and progress have been made, it is estimated that less than $15 \%$ of the world's population is covered by quality cancer registration $[7,43,44,50]$. 


\section{A brief history of cancer registration in Romania}

In Romania, mandatory cancer registration and reporting has been set up in 1981, following the issue of the Ministry of Health (MS) Order No. 219 of 1980 [51]. The order was aimed at setting up Territorial Cancer Registers in each of the 41 counties and 6 sectors in Bucharest [51]. The data collected at this level were centralized annually at the Center for Statistics and Medical Documentation of the Ministry of Public Health (MS Order No. 219 of 1980). The order was abrogated in 2002 by the Ministry of Health and Family Order no. 871/2002 that provided an update of the legislative framework of cancer registration aimed at improving compliance for cancer registration [51]. Even though the legislative framework was established there has been no functioning population-based cancer registration during until 2007, except the Cluj Cancer Registry. Cancer registration in Romania is currently legislated by the legal frame provided by the Order of the Ministry of Public Health number 2027/Nov 2007, which was aimed at implementing the recommendations of the European Network of Cancer Registries (ENCR) in Romania [51]. The order approved the setting up of eight regional cancer registries covering the entire country's territory [7,51]. To our best knowledge, at the moment, only two cancer registries are affiliated the the ENCR: The North-Western Regional Cancer Registry (NWRCR) and the West Regional Cancer Registry [52]. The data sources for the NWRCR are cancer notifications, pathology reports and death certificates. The cancer notification is a standard paper form used throughout Romania. These forms are received from all hospitals in the North-Western Region, except for "Prof. Dr. Ion Chiricuţă" Oncology Institute in Cluj-Napoca (IOCN), which submits electronic notifications from the Institutional Cancer Registry of IOCN [51].

Since 2008 the NWRCR has produced periodical reports on cancer incidence, prevalence and mortality in the NW region of Romania, the latest published in 2016 covering the year 2012 [51]. The data contained in the NWRCR have represented a valuable source for population-based epidemiological studies in the field of oncology [53-56]. The NWRCR is also a data source for the GLOBOCAN project [57]. Since 2016 the Electronic Online Notifications were implemented on the NWRCR, thanks to a European Economic Area Grant. The activity of the cancer registries observe protection of personal data and is consistent with national legislation (the law 677/2001) regarding the protecting of personal data and from the 25th of May 2018 is also subjected to the General Data Protection Regulation and with the European Network of Cancer Registries guidelines [20,51,58]. Unfortunately, according to Forsea, Romania is one of only nine European countries for which the latest national incidence data are just estimated using partial registration data available and data from registries in neighboring countries [7].

\section{Progress in the field and future challenges}

The registry results produced by population-based registries have evolved from calculating basic epidemiological data (incidence, prevalence, mortality) to diverse applications in disease prevention, early diagnosis and screening programs, treatment response, health care planning, decision making and disease control programs $[2,4]$.

In order to have comparable data across various geographical regions several study groups have proposed guidelines and recommendations on registry development, implementation, operation and evaluation [2]. The guidelines provide case definitions, patient outcomes definitions, mandatory and optional datasets $[2,4]$. The usage of international standard coding systems has improved consistency, comparability, data exchange and reuse [15]. Efforts such as the epSOS project which was aimed to "develop, pilot and evaluate cross-border eHealth services and to formulate recommendations for future work", are cross-border initiatives that are currently being continued by several other work groups $[15,59,60]$. The value of such projects resides in the fact that the data generated can be reused by other registries [15]. In recent years, several such common datasets have been produced in the EU region [15].

The expansion of the population covered by registries is one future challenge in this field [4]. The common dataset approach seems even more valid in the current migrational global context, due to the risk of losing patients from observation [4]. Migration also affects the result of medical registries due to the impact the demographic data of a geographically defined target population (commonly used for population- based registries).

In certain areas registries can and should be part of disease control programs. According to Parkin populationbased cancer registries are "an essential component of a fully developed cancer-control programs" [4]. The results of medical registries can also be used to assess the efficiency of screening programs or can include the patients that have participated in a cancer screening program $[61,62]$.

Another future direction for medical registries is represented by the aim of increasing patient involvement. At present patients are not involved as a rule in the design phase of the registry and usually have limited access to their personal collected data [63]. The involvement of patients even in the data collection process could improve the patient perception on medical registries and data collection, by the addition of variables that matter to the registered individuals [63]. Also, for certain domains, like patient care, subjects involvement can be translated into a gain in the capacity to collect support care data in real time [63].

The patient's perception on data collection can favor medical registries in the delicate balance between the right to privacy and the need for information in the public health domain. The evolution of legal frameworks 
for patient registration had a major impact on the results of medical registries. In some fields, like cancer registration, the implementation of mandatory reporting of cancer cases, in some regions of the world, and the implementation of a legal framework that exempts cancer registries from obtaining informed consent from registered cases, in the setting of public health-related data collection, has improved the registries coverage and results [4].

Electronic data collection also had a major impact on the results of medical registries. Although paper-based data collection may now be seen as a historical method, in 2014 half of the EU registries were still gathering data based on paper-based documents (questionnaires, health records and laboratory results) [15]. On the other hand, due to the strict dataset definition of medical registries, even in the setting of "high quality" EHRs that allow partial automatization of registration activity, the workload is still high [64].

In recent years a growing interest for the implementation of randomized controlled trials using patient registries has been observed [6]. Registries, in this setting, can serve as platforms for rapid, consecutive, patient enrolment and recruitment and trial operationalization [6]. This approach has a potential for achieving completeness of follow-up, cost reduction and generalization of the results [6]. Linking biobanks with high quality registry data can open new research in frontier domains like genomics and epidemiology [65]. In some countries like Sweden, for example, a Biobank Register has been implemented [66].

Increasingly, data from population registers are linked to administrative databases on the provision of health services, thus allowing for the evaluation of performance indicators to monitor the health system in a particular geographic area. Adding prognostic indicators to the population registry data set is important for such analyses and is facilitated by the introduction of structured medical reports. Health service assessors use registry survival data to evaluate service performance. Conversely, researchers who analyze data from registries can point out the existence of developments that are of concern to health authorities, for example Fetica et al have noted a much lower survival for women with follicular lymphoma in Cluj County, Romania [54].

Medical registries have potential to evolve to a next generation by taking benefit from recent semantic web technology developments. Up to now information technology has offered many solutions to collect, validate and analyze data. Structuring, coding and integration of eclectic data from diverse sources are challenges that a registry starts to face, and ontology-based approaches become more and more appealing. Novel solutions using Web Ontology Language (OWL), a Semantic Web language aimed at representing rich and complex knowledge on different subjects by using their characteristics and their interactions, have emerged [67, 68]. Ontologies constitute a standard mechanism for representing knowledge and are translated into formal representations such as, for example, OWL. Modern technologies implemented by the Semantic Web are being used more and more to represent the course of the disease in an automated form [69]. There is an increasing need for multi-sourced widely shareable registry data. The next generation registry will shift the database-centered thinking to a focus on medical concepts and their relations, from surveillance to improve clinical care in real time and integration in a "big data" health information system [70].

\section{Conclusions}

Medical registries have constantly evolved in the past century, allowing access to quality, non-biased data, that can reliably be used for a multitude of purposes that span from general medical practice to research and policies making. The extended use of medical registries has driven constant improvement of data and procedure quality. In everyday medical practice the impact of medical registries provide practitioners with relevant, "real-world", information on disease evolution in the general population and the quality of care, allowing informed decision making for the patients benefit. Efforts in providing standards for classification, coding and consistent definitions such as those used by cancer registries support the idea that widely shareable registry data can be achieved.

\section{References}

1. Solomon DJ, Henry RC, Hogan JG, Van Amburg GH, Taylor J. Evaluation and implementation of public health registries. Public Health Rep. 1991;106:142-150.

2. Gliklich RE, Dreyer NA, Leavy MB. Registries for evaluating patient outcomes: a user's guide: Government Printing Office; 2014.

3. Maret-Ouda J, Tao W, Wahlin K, Lagergren J. Nordic registrybased cohort studies: Possibilities and pitfalls when combining Nordic registry data. Scand J Public Health. 2017;45(17_ suppl):14-19.

4. Parkin DM. The evolution of the population-based cancer registry. Nat Rev Cancer. 2006;6:603-612.

5. Schmidt M, Schmidt SA, Sandegaard JL, Ehrenstein V, Pedersen L, Sørensen HT. The Danish National Patient Registry: a review of content, data quality, and research potential. Clin Epidemiol. 2015;7:449-490.

6. Li G, Sajobi TT, Menon BK, Korngut L, Lowerison M, James $\mathrm{M}$, et al. Registry-based randomized controlled trials- what are the advantages, challenges, and areas for future research? J Clin Epidemiol. 2016;80:16-24.

7. Forsea AM. Cancer registries in Europe-going forward is the only option. Ecancermedicalscience. 2016;10:641.

8. Meima A, Irgens LM, van Oortmarssen GJ, Richardus JH, Habbema JD. Disappearance of leprosy from Norway: an exploration of critical factors using an epidemiological modelling approach. Int J Epidemiol. 2002;31:991-1000.

9. Newton J, Garner S. Disease registers in England. Institute of Health Sciences, University of Oxford. 2002.

10. Pryor DB, Califf RM, Harrell FE Jr, Hlatky MA, Lee KL, Mark DB, et al. Clinical data bases. Accomplishments and unrealized potential. Med Care. 1985;23:623-647. 
11. Donaldson L. Registering a need. BMJ. 1992;305:597-598.

12. Brooke EM. The current and future use of registers in health information systems. WHO, Geneva, 1974 Available at: http:// apps.who.int/iris/bitstream/10665/36936/1/WHO_OFFSET_8.pdf. 13. World Health Organization. Electronic health records: manual for developing countries. Manila: WHO Regional Office for the Western Pacific; 2006.

14. Polygenis D. ISPOR Taxonomy of Patient Registries: Classification, Characteristics and Terms: ISPOR; 2013.

15. Zaletel M, Kralj M, Magajne M, Doupi P. Methodological Guidelines and Recommendations for Efficient and Rationale Governance of Patient Registries: Metka Zalatel. The European Journal of Public Health. 2015;25(suppl_3):ckv169. 006.

16. Mathis S, Wild C. Register für klinische und gesundheitsökonomische Fragestellungen: Einsatzbereiche von kardiovaskulären, wirbelsäulenspezifischen und neurologischen Registern und Good Practice Strategien für die Arbeit mit Registern,\# 11 HP. Wien: Editor. 2008.

17. Gladman DD, Menter A. Introduction/overview on clinical registries. Ann Rheum Dis. 2005;64 Suppl 2:ii101-ii102.

18. Stausberg J, Altmann U, Antony G, Drepper J, Sax U, Schütt A. Registers for Networked Medical Research in Germany: Situation and prospects. Applied clinical informatics. 2010;1:408-418.

19. Pedersen E. Some uses of the cancer registry in cancer control. Br J Prev Soc Med. 1962;16:105-110.

20. Data protection [Internet]. European Commission - European Commission. 2018.

21. He S, Ganzinger M, Knaup P. The intellectual property management for data sharing in a German liver cancer research network. Stud Health Technol Inform. 2012;180:891-895.

22. Hickey GL, Grant SW, Cosgriff R, Dimarakis I, Pagano D, Kappetein AP, et al. Clinical registries: governance, management, analysis and applications. Eur J Cardiothorac Surg. 2013;44:605614.

23. Dawson A, Ferrero M. Chronic Disease Management Registers: proceedings of a workshop: HM Stationery Office; 1996.

24. Levine MN, Julian JA. Registries that show efficacy: good, but not good enough. J Clin Oncol. 2008;26:5316-5319.

25. Jensen OM. Cancer registration: principles and methods: IARC; 1991.

26. Bray F, Znaor A, Cueva P, Korir A, Swaminathan R, Ullrich A, et al. Planning and developing population-based cancer registration in low-and middle-income settings: IARC (International Agency for Research on Cancer); 2014.

27. Arts DG, De Keizer NF, Scheffer GJ. Defining and improving data quality in medical registries: a literature review, case study, and generic framework. J Am Med Inform Assoc. 2002;9:600-611.

28. Bray F, Parkin DM. Evaluation of data quality in the cancer registry: principles and methods. Part I: comparability, validity and timeliness. Eur J Cancer. 2009;45:747-755.

29. Parkin DM, Bray F. Evaluation of data quality in the cancer registry: principles and methods Part II. Completeness. Eur J Cancer. 2009;45:756-764.

30. Pass HI. Medical registries: continued attempts for robust quality data. J Thorac Oncol. 2010;5(6 Suppl 2):S198-S199.

31. van der Putten E, van der Velden JW, Siers A, Hamersma EA. A pilot study on the quality of data management in a cancer clinical trial. Control Clin Trials. 1987;8:96-100.

32. Sorensen HT, Sabroe S, Olsen J. A framework for evaluation of secondary data sources for epidemiological research. Int $\mathrm{J}$
Epidemiol. 1996;25:435-442.

33. Drolet BC, Johnson KB. Categorizing the world of registries. J Biomed Inform. 2008;41:1009-1020.

34. Irgens LM, Bjerkedal T. Epidemiology of leprosy in Norway: the history of The National Leprosy Registry of Norway from 1856 until today. Int J Epidemiol. 1973;2:81-89.

35. Irgens LM. The roots of Norwegian epidemiology-Norwegian epidemiology in the 19th century. Norsk epidemiologi. 2015;25.

36. Bryder L. 'Not always one and the same thing': the registration of tuberculosis deaths in Britain, 1900-1950. Soc Hist Med. 1996;9:253-265.

37. Dye C. Global epidemiology of tuberculosis. Lancet. 2006;367:938-940.

38. Van Hest NA, Story A, Grant AD, Antoine D, Crofts JP, Watson JM. Record-linkage and capture-recapture analysis to estimate the incidence and completeness of reporting of tuberculosis in England 1999-2002. Epidemiol Infect. 2008;136:1606-1616.

39. Glaziou P, Falzon D, Floyd K, Raviglione M, editors. Global epidemiology of tuberculosis. Seminars in respiratory and critical care medicine.2013;34:3-16 .

40. World Health Organization. Global tuberculosis report 2012. Geneva, Switzerland: WHO; 2012.

41. Rare Disease Registries in Europe. In: Orphanet, editor. Orphanet Report Series Rare Diseases collection.

42. World Health Organization. Global report on diabetes: World Health Organization; 2016.

43. Bray F, Ferlay J, Laversanne M, Brewster DH, Gombe Mbalawa C, Kohler B, et al. Cancer Incidence in Five Continents: Inclusion criteria, highlights from Volume $\mathrm{X}$ and the global status of cancer registration. Int J Cancer. 2015;137:2060-2071.

44. Forman D BF, Brewster DH, et al. Cancer incidence in five continents, Volume X. Lyon; Geneva: International Agency for Research on Cancer; Distributed by WHO Press, World Health Organization, 2014.

45. Wagner G. History of cancer registration. Cancer registration: principles and methods IARC Scientific Publications. 1991:3-6.

46. Gjerstorff ML. The Danish Cancer Registry. Scand J Public Health. 2011;39(7 Suppl):42-45.

47. Barlow L, Westergren K, Holmberg L, Talbäck M. The completeness of the Swedish Cancer Register: a sample survey for year 1998. Acta Oncol. 2009;48:27-33.

48. Navarro C, Martos C, Ardanaz E, Galceran J, Izarzugaza I, Peris-Bonet R, et al. Population-based cancer registries in Spain and their role in cancer control. Ann Oncol. 2010;21 Suppl 3:iii3-iii13. 49. Stocks P. Cancer registration and studies of incidence by surveys. Bull World Health Organ. 1959;20:697-715.

50. Beaulieu N, Bloom D, Bloom R, Stein R. Breakaway: The global burden of cancer-challenges and opportunities. The Economist Intelligence Unit. 2009.

51. Coza D, Şuteu O, Blaga M, Nicula F, Moldovan A, AchimasCadariu P, et al. Cancer report in North-Western Region of Romania 2012: incidence, mortality, survival, and prevalence. Cluj-Napoca: Casa Cărţii de Ştiinţă; 2016.

52. The European Network of Cancer Registries. ENCR Members contact list 2018 .

53. Şuteu O, Blaga ML, Nicula F, Şuteu P, Coza O, AchimaşCadariu $\mathrm{P}$, et al. Incidence trends and survival of skin melanoma and squamous cell carcinoma in Cluj County, Romania. Eur J Cancer Prev. 2017 Sep;26 Joining forces for better cancer registration in Europe:S176-S182.

54. Fetica B, Achimas-Cadariu P, Pop B, Dima D, Petrov L, Perry 
AM, et al. Non-Hodgkin lymphoma in Romania: a single-centre experience. Hematol Oncol. 2017;35:198-205.

55. Georgakis MK, Dessypris N, Baka M, Moschovi M, Papadakis $\mathrm{V}$, Polychronopoulou S, et al. Neuroblastoma among children in Southern and Eastern European cancer registries: Variations in incidence and temporal trends compared to US. Int J Cancer. 2018;142:1977-1985.

56. Georgakis MK, Papathoma P, Ryzhov A, Zivkovic-Perisic S, Eser S, Taraszkiewicz $Ł$, et al. Malignant central nervous system tumors among adolescents and young adults (15-39 years old) in 14 Southern-Eastern European registries and the US Surveillance, Epidemiology, and End Results program: Mortality and survival patterns. Cancer. 2017;123:4458-4471.

57. Ferlay J, Steliarova-Foucher E, Lortet-Tieulent J, Rosso S, Coebergh JW, Comber H, et al. Cancer incidence and mortality patterns in Europe: estimates for 40 countries in 2012. Eur J Cancer. 2013;49:1374-1403.

58. Tyczynski JE, Démaret D, Parkin DM, editors. Standards and guidelines for cancer registration in Europe: the ENCR recommendations: International Agency for Research on Cancer; 2003.

59. Moharra M, Almazán C, Decool M, Nilsson AL, Allegretti N, Seven M. Implementation of a cross-border health service: physician and pharmacists' opinions from the epSOS project. Fam Pract. 2015;32:564-567.

60. Digital Single Market. Cross-border health project epSOS: What has it achieved?

61. Májek O, Dušková J, Dvořák V, Beková A, Klimeš D, Blaha $\mathrm{M}$, et al. Performance indicators in a newly established organized cervical screening programme: registry-based analysis in the Czech Republic. Eur J Cancer Prev. 2017;26:232-239.
62. Elfström KM, Sparén P, Olausson P, Almstedt P, Strander B, Dillner J. Registry-based assessment of the status of cervical screening in Sweden. J Med Screen. 2016;23:217-226.

63. Nelson EC, Dixon-Woods M, Batalden PB, Homa K, Van Citters AD, Morgan TS, et al. Patient focused registries can improve health, care, and science. BMJ. 2016;354:i3319.

64. Coorevits P, Sundgren M, Klein GO, Bahr A, Claerhout B, Daniel C, et al. Electronic health records: new opportunities for clinical research. J Intern Med. 2013;274:547-560.

65. Leitsalu L, Alavere H, Tammesoo ML, Leego E, Metspalu A. Linking a population biobank with national health registries-the Estonian experience. J Pers Med. 2015;5:96-106.

66. Norlin L, Fransson M, Eaker S, Elinder G, Litton J-E. Adapting research to the 21 st century-the Swedish Biobank Register. Norsk epidemiologi. 2012;21.

67. González-Beltrán A, Finkelstein A, Wilkinson JM, Kramer J. Domain Concept-Based Queries for Cancer Research Data Sources. 22nd IEEE International Symposium on ComputerBased Medical Systems (CBMS' 09), Volume 0. Albuquerque, New Mexico, USA: IEEE Computer Society; 2009.

68. Bianconi F, Brunori V, Valigi P, Rosa FL, Stracci F. Information Technology as Tools for Cancer Registry and Regional Cancer Network Integration. IEEE Transactions on Systems, Man, and Cybernetics - Part A: Systems and Humans. 2012;42:1410-24.

69. Esteban-Gil A, Fernández-Breis JT, Boeker M. Analysis and visualization of disease courses in a semantically-enabled cancer registry. J Biomed Semantics. 2017;8:46.

70. Mate S, Köpcke F, Toddenroth D, Martin M, Prokosch HU, Bürkle T, et al. Ontology-based data integration between clinical and research systems. PLoS One. 2015;10:e0116656. 